\title{
Impact of Selected Chemical Characteristics of Cold-Pressed Oils on their Oxidative Stability Determined Using the Rancimat and Pressure Differential Scanning Calorimetry Method
}

\author{
Edyta Symoniuk $^{1}$ (D) Katarzyna Ratusz $^{1} \cdot$ Ewa Ostrowska-Ligęza $^{2} \cdot$ Krzysztof Krygier $^{1}$
}

Received: 16 March 2017 / Accepted: 27 October 2017 / Published online: 7 November 2017

(C) The Author(s) 2017. This article is an open access publication

\begin{abstract}
In this study, 27 market and edible cold-pressed oils from 10 different oilseeds were analysed. Oxidative stability and the chemical composition of oils were evaluated. The oils were investigated for their primary quality, fatty acid composition, total phenolic content and antioxidant activity. Rancimat and pressure differential scanning calorimetry (PDSC) were used to assess oils oxidative stability. Principal component analysis (PCA) was conducted to determinate impact of selected chemical characteristics on tested oils' oxidative stability in accelerated modes. PCA indicated that none of the chemical compounds correlated strongly with the oils' oxidative stability determined by the Rancimat method. Correlation coefficients describing the impact of different chemical compounds on induction time determined using the Rancimat method were between $r=-0.54$ (C18:3) to $r=0.62$ (chlorophyll pigments). Oxidative stability of oils determined using the Rancimat and pressure differential scanning calorimetry (PDSC) were characterised by low correlation $(r=0.66)$. According to the statistical analyses, oils were divided into four groups, which depend on the method of oxidative stability evaluation did not differ.
\end{abstract}

Keywords Cold-pressed oils · Correlation coefficient . Oxidative stability $\cdot$ PCA $\cdot$ PDSC $\cdot$ Rancimat

Edyta Symoniuk

edyta_popis@sggw.pl

1 Department of Food Technology, Faculty of Food Science, Warsaw University of Life Sciences, Nowoursynowska St. 166, 02-787 Warsaw, Poland

2 Department of Food Chemistry, Faculty of Food Science, Warsaw University of Life Sciences, Warsaw, Poland

\section{Introduction}

Oxidative stability is one of the most important parameters used to assess oil quality, determining its resistance to the oxidation process. Oxidation occurs in unsaturated fatty acids during oil storage or heat treatment and causes their quality deterioration. Particularly easily oxidised are oils having a high content of polyunsaturated fatty acids, especially linolenic acid. Oils obtained by cold pressed technology in addition to triacylglycerides also contained lipidaccompanying compounds. Therefore, its stability depends not only on the fatty acids composition but also on the content of antioxidants, primary and secondary oxidation products, metals and other contaminants which might accelerate or inhibit oxidation process (Choe and Min 2006; Górnaś et al. 2014; Szterk et al. 2010).

Edible oils' oxidative stability might be determined using different methods. One of the most popular is the shelf-life test run under real state conditions. Shelf-life test involves placing oil samples on shelves and evaluating its basic quality features at regular time intervals (Farhoosh 2007). Nevertheless, due to the long duration of the test (several months) and the need to apply chemical reagents currently, to determinate oil oxidative stability, accelerated methods are mainly used. The Rancimat test is a popularly used accelerated oxidative stability evaluation method, it allows for a faster determination of the stability time by submitting the oil sample to a high temperature and constant airflow. Oxidative stability in the Rancimat test is referred as the induction time or oxidative stability index (OSI). Induction time corresponds to a sudden growth in the water conductivity in measuring vessel. Water conductivity is dependent on volatile compounds formed from the decomposition of unstable peroxides produced during the first step of oxidation (Raczyk et al. 2016; Shahidi and Zhong 2005). 
Oil oxidative stability can also be measured using thermal technics. The pressure differential scanning calorimetry (PDSC) is a useful way of measuring the oxidative stability of vegetable oils in an accelerated mode; results are obtained faster due to the use of high temperature and pressure. PDSC method uses the fact that oil oxidation is an exothermic process. A heat released during the oxidation of oil sample is compared with a reference sample. The difference in generated heat is recorded over time as a graph from which can be the determined onset time $\left(\tau_{\mathrm{on}}\right)$ and the maximum of oxidation time $\left(\tau_{\max }\right)$. Both parameters are used to characterise oil oxidation process, but they correspond with different stages of oxidation (Ciemniewska-Żytkiewicz et al. 2014; Kowalski et al. 2004).

The products formed in the oxidation process have an adverse effect on the human body; therefore, proper assessment of the oxidative stability is a decisive step in the safety assessment of oil. Available methods used for evaluating oils' oxidative stability are very diverse. The literature presents a number of differences between the Rancimat and differential scanning calorimetry methods (Šimon et al. 2000; Woo et al. 2001). Available studies show that oxidative induction time data determined using these two methods might be divergent. This may be related to different oxygen diffusion within the samples depending on the atmosphere (oxygen, oxygen) in which the oxidation reaction takes place (Šimon and Kolman 2001). According to the state of our knowledge, comparison of the Rancimat and PDSC induction time of coldpressed oils obtained from different oilseeds was not investigated by other researchers. In the present study, the oxidative stability of various cold-pressed oils was evaluated using a standard Rancimat method and pressure differential scanning calorimetry. Furthermore, oils were examined to their chemical composition. Results were compared and subjected to principal component analyses.

\section{Materials and Methods}

\section{Oil Samples}

Research material consists of 27 cold-pressed oil samples: linseed (Linum usitatissimum L.) (LO, $n=5$ ), rapeseed (Brassica napus L. var. napus) (RO, $n=5$ ), camelina (Camelina sativa L.) $(\mathrm{CO}, n=3)$, black cumin (Nigella sativa L.) (NO, $n=2$ ), evening primrose (Oenothera L.) (EPO, $n=2)$, hempseed (Cannabis sativa L.) (HO, $n=2)$, milk thistle (Silybum marianum) (MTO, $n=2$ ), poppy (Papaver dubium) (PO, $n=2)$, pumpkin (Cucurbita pepo) (PUO, $n=2)$ and sunflower (Helianthus L.) (SO, $n=2)$ oil. All oils came from Polish manufacturers and were produced and purchased during their shelf life on the Polish market.

\section{Chemicals}

All reagents and solvents used for the analyses and sample preparation for $\mathrm{GC}$ were obtained from POCH S.A. (Gliwice, Poland). The Food Industry 37 Component Fatty Acid Methyl Esters (FAME) mix standard was supplied by Restek (Bellefonte, PA, US). Other reagents used for spectrophotometric analyses of analytical grade were from Sigma-Aldrich (Poznań, Poland).

Spectrophotometric measurements were taken using Helios Gamma UV-Vis spectrophotometer (Loughborough, England) with an optical glass cuvette and applying 10-mm optical path length.

\section{Acid, Peroxide, $p$-Anisidine Value and Total Oxidation Indicator Determination}

The acid value (AV) was determined according to the American Oil Chemists' Society (AOCS) Official Method Cd 3d-63 (2000). Determination of the peroxide value (PV) was made by applying the AOCS Official Method 965.33 (1999), and the content of secondary oxidation products known as $p$-anisidine value ( $p$-AnV) was determined by the AOCS Official Method Cd 18-90 (2002). Additionally, the overall sample rate of oxidation known as total oxidation (Totox) indicator (Totox $=2 \mathrm{PV}+p-\mathrm{AnV}$ ) was calculated.

\section{Chlorophyll and Carotenoid Pigments Content}

The chlorophyll content was determined according to the AOCS Official Method Cc 13i-96 (1998). The result of measurement was expressed in $\mathrm{mg}$ of pheophytin $\mathrm{kg}^{-1}$. Carotenoid content was expressed as $\beta$-carotene in $\mathrm{mg}$ per $\mathrm{kg}$ of oil. The measurement was done according to BS 684 (1977) Section 2.20.

\section{Analysis of Fatty Acid Composition}

Cold-pressed oils were prepared for the GC-flame ionisation detector (FID) analysis as fatty acid methyl esters according to the AOCS Official Method Ce 2-66 (1997). Determination of esterified fatty acids in tested oils was done according to the AOAC Official Method 996.06 (2001) with modifications described by Symoniuk et al. (2016).

Methyl esters were analysed on a TRACE 1300 (Thermo Fisher Scientific, Inc., Wilmington, DE, US) gas chromatograph equipped with Restek BPX70 GC capillary column (60 $\mathrm{m}$ in length $\times 0.22 \mathrm{~mm}$ I.D., $0.25 \mu \mathrm{m}$ of film thickness) and flame ionisation detector (FID). The fatty acid composition of analysed samples was presented as percentage participation in total fatty acid composition. 


\section{Calculated Oxidizability Value}

The calculated oxidizability (Cox) value of the cold-pressed oils was calculated according to the Fatemi and Hammond (1980) formula:

Cox value $=\left\{[C 18: 1(\%)]+10.3^{*}[C 18: 2(\%)]+21.6^{*}[C 18: 3(\%)]\right\} / 100$

\section{Total Free Radical Scavenger Capacity Using 2, 2-Diphenyl-1-Picrylhydrazyl Radical}

Determination of the 2,2-diphenyl-1-pikrylohydrazylowych $\left(\mathrm{DPPH}^{*}\right)$ free radical binding capacity was determinate according to the Espin et al. (2000) method. Tested oil antioxidant potential was characterised by the antiradical efficiency; the result was expressed as Trolox equivalent antioxidant capacity (TEAC) (Sánchez-Moreno et al. 1998). Oil solutions in dichloromethane with a concentration of $2 \%$ oil were prepared. The analytical sample consisted of $3 \mathrm{~cm}^{3}$ of an oil solution, $1 \mathrm{~cm}^{3}$ of methanol and $1 \mathrm{~cm}^{3}$ of the solution of $0.5 \mathrm{mM}$ of $\mathrm{DPPH}^{*}$ in methanol. Absorbance was measured at 1-min intervals for $60 \mathrm{~min}$ at wavelength $\lambda=517 \mathrm{~nm}$. Nil consisted of $3 \mathrm{~cm}^{3}$ of dichloromethane, $1 \mathrm{~cm}^{3}$ of methanol and $1 \mathrm{~cm}^{3}$ of DPPH ${ }^{*}$ free radical solution. Calibration was done using a mixture of $3 \mathrm{~cm}^{3}$ of dichloromethane and $2 \mathrm{~cm}^{3}$ of methanol. Radical scavenger capacity was calculated based on a standard curve prepared with Trolox standard solutions.

\section{Total Phenolic Content}

Total phenolic content (TPC) of cold-pressed oils was determined using the Folin-Ciocalteu reagent according with Siger et al. (2008) method. Sample preparation based on a transfer of $2 \mathrm{~mL}$ of oil extract and $0.5 \mathrm{~mL}$ of Folin-Ciocalteu to a 10 -mL flask. Next sample was mixed using a vortex and allowed to stand for $3 \mathrm{~min}$. After $3 \mathrm{~min}, 1 \mathrm{~mL}$ of saturated sodium carbonate solution was added and made up with distilled water to $10 \mathrm{~mL}$. The absorbance of all samples was measured at $725 \mathrm{~nm}$ after $1 \mathrm{~h}$, compared to control sample. Total phenolic content was calculated using a standard curve prepared with ferulic acid (FEA).

\section{Oxidative Stability—Rancimat Measurement}

Rancimat 743 Metrohm apparatus (Herisau, Switzerland) was used to determine the oxidative stability of oils. The outcome measure was the induction time. Measurements were done according to ISO 6886:2009. Oil sample of $2.5 \pm 0.01 \mathrm{~g}$ was oxidised with a heating temperature of $100{ }^{\circ} \mathrm{C}$ and an air flow of $20 \mathrm{~L} \mathrm{~h}^{-1}$. Volatile products formed from oxidation reaction were soluble in $0.06 \mathrm{~L}$ of distilled water. The induction time was recorded automatically by apparatus software with the accuracy of 0.01 ; the result was expressed in hours.

\section{Oxidative Stability—Pressure Differential Scanning Calorimetry Isothermal Measurement}

The oxidative stability of tested oils was determined using a DSC (Q20, TA Instruments) coupled with a high-pressure cell (Q20P). Before measurement, the instrument was calibrated using standard, high-purity indium. Calibration of the PDSC apparatus consisted in determining the transition temperature and enthalpy of indium $\left(156.6^{\circ} \mathrm{C}, 28.57 \mathrm{~J} \mathrm{~g}^{-1}\right)$ in an oxygen atmosphere, at a rate heating rate of $5^{\circ} \mathrm{C} \mathrm{min}^{-1}$. Oil sample of 3-4 mg of oil was oxidised into open aluminium pan. Oxidation was determined under isothermal conditions, at a temperature of $120^{\circ} \mathrm{C}$ and pressure of $1380-1400 \mathrm{kPa} \mathrm{O}_{2}$.

After the maximum of heat flow was reached, the experiments were manually stopped. Analysis of diagrams was performed using TA Universal Analysis 2000 software. The oxidation induction time was determined based on the onset time of oxidation with the accuracy of 0.01 . The result of PDSC measurement was expressed in minutes.

\section{Statistics Analyses}

Measurements were done in triplicate; results in tables are the average of replications with deviation standard. Results were subjected to analysis of variance (ANOVA). Significant means were subjected to analysis by post hoc Tuckey's multiple range test $(\alpha=0.05)$. Pearson correlation coefficients, principal component analysis (PCA) and agglomerative hierarchical clustering (AHC) were performed using XLSTAT (2017, Addinsoft, Inc., Brooklyn, NY, USA).

\section{Results and Discussion}

\section{Chemical Composition of Analysed Oils}

The results of oils acid, peroxide, $p$-anisidine values and Totox indicator are summarised in Table 1. According to the Codex Alimentarius standard, analysed oils were of good quality. Oils' hydrolysis degree $\left(<4 \mathrm{mg} \mathrm{KOH} \mathrm{g}^{-1}\right)$ and oxidation degree $\left(15 \mathrm{mEq} \mathrm{O} \mathrm{kg}^{-1}\right)$ not exceed the recommended limits for cold-pressed oils. Determined $p$-anisidine value, which reflects the content of lipids secondary oxidation products, was low. Analysed oils peroxide value was higher than $p$ anisidine value, which is typical for cold-pressed oils.

Oils were also examined to chlorophyll and carotenoid pigments content; results are presented in Table 1. Both chlorophyll and carotenoid pigments found in the cold-pressed oils affect their oxidative stability (Kamal-Eldin 2006). It is preferable to avoid a high amount of chlorophyll pigments as they 
Table 1 Selected chemical characteristics of analysed cold-pressed oils

\begin{tabular}{|c|c|c|c|c|c|c|c|c|}
\hline Oil & 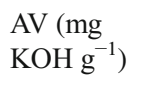 & $\begin{array}{l}\mathrm{PV}(\mathrm{mg} \\
\left.\mathrm{O}_{2} \mathrm{~kg}^{-1}\right)\end{array}$ & $p-\mathrm{AnV}$ & $\begin{array}{l}\text { Totox } \\
\text { indicator }\end{array}$ & $\begin{array}{l}\text { Chlorophylls (mg of } \\
\text { pheophytin } \mathrm{kg}^{-1} \text { oil) }\end{array}$ & $\begin{array}{l}\text { Carotenoids (mg of } \\
\beta \text {-carotene } \mathrm{kg}^{-1} \text { oil) }\end{array}$ & $\begin{array}{l}\text { Trolox equivalent } \\
\left(\mathrm{mM} \mathrm{kg}^{-1} \text { oil) }\right.\end{array}$ & 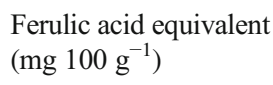 \\
\hline LO1 & $0.52^{\mathrm{ijk} 1}$ & $2.03^{\mathrm{h}}$ & $0.51^{\mathrm{cd}}$ & $4.57^{\mathrm{j}}$ & $0.06^{\mathrm{a}}$ & $34.07^{\mathrm{n}}$ & $2.01^{\mathrm{j}}$ & $84.5^{\mathrm{hi}}$ \\
\hline LO2 & $0.21^{\mathrm{a}}$ & $1.57^{\mathrm{e}}$ & $0.23^{\mathrm{a}}$ & $3.37^{\mathrm{e}}$ & $0.11^{\mathrm{ab}}$ & $34.21^{\mathrm{n}}$ & $1.99^{\mathrm{i}}$ & $80.2^{\text {ghi }}$ \\
\hline LO3 & $0.40^{\mathrm{defg}}$ & $1.80^{\mathrm{f}}$ & $0.89^{\mathrm{ij}}$ & $4.49^{\mathrm{ij}}$ & $0.15^{\mathrm{ab}}$ & $34.97^{\circ}$ & $2.01^{\mathrm{j}}$ & $89.6^{\mathrm{ij}}$ \\
\hline LO4 & $0.53^{\mathrm{ijk} 1}$ & $2.00^{\mathrm{gh}}$ & $1.04^{\mathrm{k}}$ & $5.04^{\mathrm{kl}}$ & $0.19^{\mathrm{b}}$ & $38.75^{\mathrm{p}}$ & $1.97^{\mathrm{hi}}$ & $65.4^{\mathrm{def}}$ \\
\hline LO5 & $1.21^{\mathrm{n}}$ & $2.54^{\mathrm{j}}$ & $0.53^{\text {cde }}$ & $5.61^{\mathrm{n}}$ & $0.79^{\mathrm{d}}$ & $18.43^{1}$ & $1.98^{\mathrm{i}}$ & $60.3^{\text {cde }}$ \\
\hline RO1 & $0.51^{\mathrm{ijkl}}$ & $1.04^{\mathrm{c}}$ & $0.54^{\text {cde }}$ & $2.62^{\mathrm{bc}}$ & $2.62^{\mathrm{g}}$ & $11.52^{\mathrm{j}}$ & $1.96^{\mathrm{h}}$ & $112.8^{\mathrm{k}}$ \\
\hline $\mathrm{RO} 2$ & $0.46^{\text {fghij }}$ & $2.02^{\mathrm{h}}$ & $0.98^{\mathrm{jk}}$ & $5.02^{\mathrm{kl}}$ & $2.58^{\mathrm{g}}$ & $11.17^{\mathrm{i}}$ & $1.81^{\mathrm{ef}}$ & $80.3^{\text {ghe }}$ \\
\hline RO3 & $0.29^{\mathrm{abc}}$ & $1.56^{\mathrm{e}}$ & $0.72^{\mathrm{h}}$ & $3.84^{\mathrm{gh}}$ & $2.62^{\mathrm{g}}$ & $10.26^{\mathrm{h}}$ & $1.96^{\mathrm{h}}$ & $85.1^{\mathrm{i}}$ \\
\hline RO4 & $0.67^{\mathrm{m}}$ & $1.98^{\mathrm{gh}}$ & $0.87^{\mathrm{i}}$ & $4.83^{\mathrm{jk}}$ & $1.93^{\mathrm{f}}$ & $12.83^{\mathrm{k}}$ & $1.88^{\mathrm{g}}$ & $60.2^{\text {cde }}$ \\
\hline RO5 & $0.43^{\text {efgh }}$ & $1.23^{\mathrm{d}}$ & $0.53^{\text {cde }}$ & $2.99^{\mathrm{d}}$ & $2.02^{\mathrm{f}}$ & $11.43^{\mathrm{ij}}$ & $1.87^{\mathrm{g}}$ & $50.7^{\mathrm{abc}}$ \\
\hline $\mathrm{CO} 1$ & $0.25^{\mathrm{ab}}$ & $2.37^{\mathrm{i}}$ & $0.51^{\mathrm{cd}}$ & $5.25^{1 \mathrm{~m}}$ & $0.60^{\mathrm{c}}$ & $2.28^{\mathrm{a}}$ & $1.98^{\mathrm{i}}$ & $90.2^{\mathrm{ij}}$ \\
\hline $\mathrm{CO} 2$ & 0.38 & $2.87^{\mathrm{k}}$ & $0.67^{\mathrm{gh}}$ & $6.41^{\circ}$ & $1.34^{\mathrm{e}}$ & $8.89^{f}$ & $1.88^{\mathrm{g}}$ & $100.3^{j}$ \\
\hline $\mathrm{CO} 3$ & $0.29^{\mathrm{abc}}$ & $3.00^{\mathrm{k}}$ & $0.45^{\mathrm{bc}}$ & $6.45^{\circ}$ & $2.60^{\mathrm{g}}$ & $3.83^{\mathrm{b}}$ & $2.44^{\mathrm{n}}$ & $120.1^{\mathrm{k}}$ \\
\hline BCO1 & $0.23^{\mathrm{a}}$ & $1.03^{\mathrm{c}}$ & $0.87^{\mathrm{i}}$ & $2.93^{\mathrm{cd}}$ & $67.12^{\mathrm{p}}$ & $28.32^{\mathrm{m}}$ & $1.85^{\mathrm{fg}}$ & $115.9^{\mathrm{k}}$ \\
\hline $\mathrm{BCO} 2$ & $0.35^{\text {cde }}$ & $1.56^{\mathrm{e}}$ & $0.61^{\mathrm{efg}}$ & $3.73^{\mathrm{fg}}$ & $62.70^{\circ}$ & $40.58^{r}$ & $3.18^{\mathrm{p}}$ & $119.2^{\mathrm{k}}$ \\
\hline EPO1 & $0.54^{\mathrm{jkl}}$ & $1.06^{\mathrm{c}}$ & $0.61^{\mathrm{efg}}$ & $2.73^{\mathrm{bcd}}$ & $14.46^{\mathrm{k}}$ & $10.52^{\mathrm{h}}$ & $0.87^{\mathrm{b}}$ & $60.2^{\text {cde }}$ \\
\hline EPO2 & $0.52^{\mathrm{ijk} 1}$ & $2.98^{\mathrm{k}}$ & $0.98^{\mathrm{jk}}$ & $6.94^{\mathrm{p}}$ & $15.94^{1}$ & $6.60^{\mathrm{e}}$ & $0.55^{\mathrm{a}}$ & $70.5^{\mathrm{efg}}$ \\
\hline HO1 & $0.60^{\mathrm{lm}}$ & $1.87^{\mathrm{fg}}$ & $0.41^{\mathrm{b}}$ & $4.15^{\mathrm{hi}}$ & $3.30^{\mathrm{h}}$ & $18.26^{1}$ & $2.06^{\mathrm{k}}$ & $178.4^{1}$ \\
\hline HO1 & $0.45^{\text {fghi }}$ & $1.56^{\mathrm{e}}$ & $0.50^{\mathrm{bcd}}$ & $3.62^{\mathrm{efg}}$ & $4.02^{\mathrm{i}}$ & $12.89^{\mathrm{k}}$ & $2.07^{\mathrm{k}}$ & $91.2^{\mathrm{ef}}$ \\
\hline MTO1 & $0.32^{\text {bcd }}$ & $1.45^{\mathrm{e}}$ & $0.56^{\mathrm{def}}$ & $3.46^{\mathrm{ef}}$ & $6.46^{\mathrm{j}}$ & $9.57^{\mathrm{g}}$ & $2.56^{\mathrm{o}}$ & $45.4^{\mathrm{ab}}$ \\
\hline MTO2 & $0.22^{\mathrm{a}}$ & $1.56^{\mathrm{e}}$ & $0.65^{\mathrm{fgh}}$ & $3.77^{\mathrm{fg}}$ & $3.93^{\mathrm{i}}$ & $4.66^{\mathrm{c}}$ & $2.14^{1}$ & $50.7^{\mathrm{abc}}$ \\
\hline PO1 & $0.47^{\mathrm{hijk}}$ & $1.02^{\mathrm{c}}$ & $0.41^{\mathrm{b}}$ & $2.45^{\mathrm{b}}$ & $0.10^{\mathrm{ab}}$ & $2.17^{\mathrm{a}}$ & $1.32^{\mathrm{c}}$ & $73.1^{\mathrm{fgh}}$ \\
\hline $\mathrm{PO} 2$ & $0.35^{\text {cde }}$ & $2.03^{\mathrm{h}}$ & $0.71^{\mathrm{h}}$ & $4.77^{\mathrm{jk}}$ & $0.11^{\mathrm{ab}}$ & $2.32^{\mathrm{a}}$ & $1.54^{\mathrm{d}}$ & $65.3^{\mathrm{def}}$ \\
\hline PUO1 & $0.47^{\mathrm{hijk}}$ & $0.87^{\mathrm{b}}$ & $0.65^{\mathrm{fgh}}$ & $2.39^{\mathrm{ab}}$ & $30.57^{\mathrm{n}}$ & $142.21^{\mathrm{s}}$ & $1.95^{\mathrm{h}}$ & $55.6^{\mathrm{bcd}}$ \\
\hline PUO2 & $0.38^{\mathrm{def}}$ & $0.73^{\mathrm{a}}$ & $0.58^{\text {defg }}$ & $2.04^{\mathrm{a}}$ & $21.94^{\mathrm{m}}$ & $342.34^{t}$ & $1.96^{\mathrm{h}}$ & $41.7^{\mathrm{a}}$ \\
\hline SO1 & $0.40^{\text {defg }}$ & $1.24^{\mathrm{d}}$ & $0.45^{\mathrm{bc}}$ & $2.93^{\mathrm{cd}}$ & $0.09^{\mathrm{ab}}$ & $6.49^{\mathrm{e}}$ & $2.34^{\mathrm{m}}$ & $52.3^{\mathrm{abc}}$ \\
\hline $\mathrm{SO} 2$ & $0.59^{\mathrm{klm}}$ & $2.34^{\mathrm{i}}$ & $0.89^{\mathrm{ij}}$ & $5.57^{\mathrm{mn}}$ & $0.11^{\mathrm{ab}}$ & $5.67^{\mathrm{d}}$ & $1.76^{\mathrm{e}}$ & $43.8^{\mathrm{a}}$ \\
\hline
\end{tabular}

Means within each column with different superscript letters $(a-t)$ are significantly $(\alpha=0.05)$ different. Each value in the table represents the mean of three measurements

can accelerate oil oxidation. Chlorophyll level in edible oils should not exceed $50 \mathrm{mg} \mathrm{kg}^{-1}$ (Matthäus and Brühl 2003). Among investigated oils, the highest chlorophyll pigments content was detected in hempseed oils. A large number of chlorophylls were also found in pumpkin and black cumin seed oils. On the other hand, the lowest chlorophyll pigments content was reported for sunflower, poppy and linseed oils. Another group of cold-pressed oil pigments are carotenoids. $\beta$-Carotene is known as the most effective provitamin A, which has an antioxidant effect on oil oxidation. In contrast to chlorophylls, carotenoids are desirable because they act as a scavenger of singlet oxygen (Shahidi and Zhong 2010). As shown in Table 1 the highest content of $\beta$-carotene was reported for pumpkin seed oils. Moreover, high content of $\beta$ carotene was also detected for linseed and hempseed oils. The lowest $\beta$-carotene amount was designated for poppy seed oils and camelina oil (CO1). Other oils were divided into two groups because of their carotenoid content. The first group of oils had content of $\beta$-carotene below $10 \mathrm{mg} \mathrm{kg}^{-1}$; camelina, black cumin and milk thistle oils were included here. The second group (rapeseed and evening primrose oils) represented oils with a content of $\beta$-carotene between 10 and $20 \mathrm{mg} \mathrm{kg}^{-1}$.

Oils were also examined for their antioxidant activity using DPPH radicals; the results are summarised in Table 1 . Antioxidants have a high impact on fat oxidative stability. Obtained results showed a significant difference in the oils' ability of radical deactivation. One of the black cumin oils (BCO2) had the strongest antiradical properties (at $3.18 \mathrm{mM}$ TEAC, quenched $91.25 \%$ of DPPH*). A similar result for black cumin oil was presented by Sielicka et al. (2014) and Haron et al. (2014). Moderately high quenching of DPPH* was observed for milk thistle oils (2.14 and 2.56 at 3.18 TEAC). The similar antioxidant activity of milk thistle oil was reported by Prescha et al. (2014). Examined sunflower oils differed significantly in their ability to scavenge free 
radicals. SO1 had greater scavenging DPPH* free radicals capacity than $\mathrm{SO} 2$. According to manufacturer's declaration, $\mathrm{SO} 2$ had an additive of tocopherols mix. Oppositely to black cumin oils, the weakest radical reduction was recorded for evening primrose oils. Analysed linseed, rapeseed, camelina and pumpkin oils had similar antioxidant activity.

Phenolic compounds have the antioxidant ability due to their redox properties; they act as reducing agents, hydrogen donors and singlet oxygen quenchers. Phenols can also have a metal-chelating potential (Rice-Evans et al. 1995). The results of total phenolic content of tested oils are shown in Table 1. The total phenolic content of analysed oils varied from 41.7 to $178.4 \mathrm{mg}$ ferulic acid $100 \mathrm{~g}^{-1}$. The highest content of phenolic compounds was determined for hempseed oil (HO1). It was found that analysed pumpkin oil (PO2) had the lowest content of phenolic acids. Black cumin, camelina $(\mathrm{CO} 3)$ and rapeseed (RO1) oils had a little lower content of phenolic acids when compared to the hempseed oil. Furthermore, phenolic acids content of sunflower, milk thistle and one of the rapeseed (RO5) oils do not differ significantly regarding pumpkin oil. Poppy and evening primrose oils had similar TPC.

Fatty acid composition affects oil oxidative stability; oils with a high content of polyunsaturated fatty acids are more easily oxidised. The fatty acid composition of analysed oils is presented in Table 2. The maximum level of saturated acids, made up mainly of palmitic acid, was obtained for black cumin and pumpkin seed oils. In contrast, sunflower (SO1) and rapeseed oils were characterised by the smallest proportion of saturated fatty acids. Examined oils differed significantly in the content of unsaturated fatty acids: monounsaturated fatty acids (MUFA) and polyunsaturated fatty acids (PUFA). Oleic acid, one of the monounsaturated fatty acid, was most dominant in sunflower (SO1) and rapeseed oils, while evening primrose oils had the lowest level of this acid. One of the most

Table 2 Percentage distribution of main fatty acid in analysed cold-pressed edible oils (\%)

\begin{tabular}{|c|c|c|c|c|c|c|c|c|c|c|c|c|c|c|c|}
\hline Oil & $\mathrm{C} 16: 0$ & C18:0 & C18:1 & C18:2 & $\mathrm{C} 18: 3$ & $\mathrm{C} 20: 0$ & $\mathrm{C} 20: 1$ & $\mathrm{C} 20: 2$ & C20:4 & C22:1 & * Other & ${ }^{*} \Sigma$ SFA & ${ }^{*} \Sigma \mathrm{MUFA}$ & ${ }^{*} \Sigma$ PUFA & COX \\
\hline $\mathrm{O} 1$ & $6.01^{\mathrm{k}}$ & $3.63^{\circ}$ & $17.74^{\mathrm{i}}$ & $15.33^{b c}$ & $56.34^{\mathrm{s}}$ & $0.11^{\mathrm{a}}$ & $0.17^{\mathrm{abcd}}$ & - & - & - & $0.67^{\mathrm{f}}$ & $9.75^{\mathrm{k}}$ & $17.91^{\mathrm{f}}$ & $71.67^{\mathrm{r}}$ & $13.93^{\mathrm{w}}$ \\
\hline $\mathrm{LO} 2$ & $6.14^{1}$ & $3.75^{\mathrm{p}}$ & $17.35^{\mathrm{g}}$ & $16.20^{\mathrm{d}}$ & $56.15^{\mathrm{r}}$ & $0.15^{\mathrm{ab}}$ & $0.22^{\mathrm{de}}$ & - & - & - & $0.04^{\mathrm{a}}$ & $10.04^{1}$ & $17.57^{\mathrm{e}}$ & $72.35^{\mathrm{s}}$ & $13.97^{\mathrm{w}>}$ \\
\hline LO3 & $5.94^{\mathrm{j}}$ & $3.67^{\circ}$ & $18.05^{\mathrm{j}}$ & $15.43^{\mathrm{c}}$ & $55.90^{\mathrm{p}}$ & $0.18^{\mathrm{bc}}$ & $0.16^{\mathrm{abcd}}$ & - & - & - & $0.67^{\mathrm{f}}$ & $9.79^{\mathrm{k}}$ & $18.21^{\mathrm{g}}$ & $71.33^{\mathrm{r}}$ & $13.84^{\mathrm{u}}$ \\
\hline LO4 & $5.86^{\mathrm{i}}$ & $3.56^{\mathrm{n}}$ & $17.55^{\mathrm{h}}$ & $16.12^{\mathrm{d}}$ & $55.92^{\mathrm{p}}$ & $0.15^{\mathrm{ab}}$ & $0.20^{\text {cde }}$ & - & - & - & $0.64^{\mathrm{f}}$ & $9.57^{\mathrm{j}}$ & $17.75^{\mathrm{ef}}$ & $72.04^{\mathrm{s}}$ & $13.91^{\mathrm{w}}$ \\
\hline LO5 & $5.76^{\mathrm{h}}$ & $4.39^{\mathrm{r}}$ & $23.36^{\mathrm{m}}$ & $15.24^{\mathrm{b}}$ & $50.10^{\circ}$ & $0.14^{\mathrm{ab}}$ & $0.21^{\mathrm{de}}$ & - & - & - & $0.80^{\mathrm{g}}$ & $10.29^{\mathrm{m}}$ & $23.57^{\mathrm{j}}$ & $65.34^{\circ}$ & $12.62^{\mathrm{t}}$ \\
\hline RO1 & $4.19^{\mathrm{c}}$ & $1.75^{\mathrm{a}}$ & $63.33^{\mathrm{s}}$ & $17.47^{\mathrm{g}}$ & $9.14^{\mathrm{h}}$ & $0.65^{\mathrm{hi}}$ & $1.82^{1}$ & $0.10^{\mathrm{a}}$ & - & $0.50^{\mathrm{b}}$ & $1.05^{\mathrm{i}}$ & $6.59^{\mathrm{d}}$ & $65.65^{\mathrm{r}}$ & $26.71^{\mathrm{d}}$ & $4.41^{\mathrm{d}}$ \\
\hline RO2 & $4.13^{\mathrm{c}}$ & $1.72^{\mathrm{a}}$ & $64.56^{\mathrm{u}}$ & $17.59^{\mathrm{g}}$ & $8.66^{\mathrm{g}}$ & $0.63^{\mathrm{h}}$ & $1.42^{\mathrm{j}}$ & $0.20^{\mathrm{a}}$ & - & $0.60^{\mathrm{b}}$ & $0.49^{\mathrm{d}}$ & $6.48^{\mathrm{c}}$ & $66.58^{\mathrm{t}}$ & $26.45^{\mathrm{cd}}$ & $4.32^{\mathrm{bc}}$ \\
\hline RO3 & $4.33^{\mathrm{d}}$ & $2.02^{\mathrm{c}}$ & $64.07^{t}$ & $17.28^{\mathrm{f}}$ & $8.60^{\mathrm{g}}$ & $0.70^{\mathrm{i}}$ & $1.43^{\mathrm{j}}$ & $0.10^{\mathrm{a}}$ & - & $0.54^{\mathrm{b}}$ & $0.93^{\mathrm{h}}$ & $7.05^{\mathrm{f}}$ & $66.04^{\mathrm{s}}$ & $25.98^{\mathrm{b}}$ & $4.27^{\mathrm{c}}$ \\
\hline $\mathrm{RO} 4$ & $4.16^{\mathrm{c}}$ & $1.73^{\mathrm{a}}$ & $64.50^{\mathrm{u}}$ & $17.59^{\mathrm{g}}$ & $8.63^{\mathrm{g}}$ & $0.61^{\mathrm{h}}$ & $1.39^{\mathrm{j}}$ & $0.12^{\mathrm{a}}$ & - & $0.52^{\mathrm{b}}$ & $0.75^{\mathrm{g}}$ & $6.50^{\mathrm{cd}}$ & $66.41^{\mathrm{t}}$ & $26.34^{\mathrm{c}}$ & $4.32^{\mathrm{bc}}$ \\
\hline $\mathrm{OO} 5$ & $4.18^{\mathrm{c}}$ & $1.87^{\mathrm{b}}$ & $64.10^{t}$ & $17.54^{\mathrm{g}}$ & $9.04^{\mathrm{h}}$ & $0.65^{\mathrm{hi}}$ & $1.63^{\mathrm{k}}$ & $0.11^{\mathrm{a}}$ & - & $0.56^{\mathrm{b}}$ & $0.32^{\mathrm{cd}}$ & $6.70^{\mathrm{e}}$ & $66.29 \mathrm{st}$ & $26.69^{\mathrm{d}}$ & $4.4^{\mathrm{d}}$ \\
\hline $\mathrm{CO} 1$ & $5.42^{\mathrm{f}}$ & $2.46^{\mathrm{gh}}$ & $15.58^{\mathrm{e}}$ & $17.99^{\mathrm{h}}$ & $34.32^{\mathrm{n}}$ & $1.34^{1}$ & $14.57^{\mathrm{m}}$ & $2.04^{\mathrm{e}}$ & $1.47^{\mathrm{b}}$ & $2.74^{\mathrm{d}}$ & $2.07^{\mathrm{k}}$ & $9.22^{\mathrm{i}}$ & $32.89^{\mathrm{n}}$ & $55.82^{\mathrm{k}}$ & $9.42^{\mathrm{n}}$ \\
\hline $\mathrm{CO} 2$ & $5.12^{\mathrm{e}}$ & $2.57^{\mathrm{i}}$ & $16.15^{\mathrm{f}}$ & $16.46^{\mathrm{e}}$ & $33.93^{\mathrm{m}}$ & $1.50^{\mathrm{m}}$ & $15.82^{\mathrm{n}}$ & $1.98^{\mathrm{e}}$ & $1.47^{\mathrm{b}}$ & $2.43^{\mathrm{c}}$ & $2.57^{\mathrm{m}}$ & $9.19^{\mathrm{i}}$ & $34.40^{\circ}$ & $53.84^{\mathrm{h}}$ & $9.18^{\mathrm{m}}$ \\
\hline $\mathrm{CO} 3$ & $5.43^{\mathrm{f}}$ & $2.66^{\mathrm{j}}$ & $16.00^{\mathrm{f}}$ & $17.19^{\mathrm{f}}$ & $31.62^{1}$ & $1.61^{\mathrm{n}}$ & $16.33^{\circ}$ & $2.12^{\mathrm{e}}$ & $1.46^{\mathrm{b}}$ & $3.21^{\mathrm{e}}$ & $2.37^{1}$ & $9.70^{\mathrm{k}}$ & $35.54^{\mathrm{p}}$ & $52.39^{\mathrm{g}}$ & $8.76^{1}$ \\
\hline $3 \mathrm{CO} 1$ & $11.96^{\mathrm{u}}$ & $2.89^{\mathrm{k}}$ & $22.69^{1}$ & $57.95^{\mathrm{p}}$ & $0.61^{\mathrm{c}}$ & $0.21^{\mathrm{cd}}$ & $0.11^{\mathrm{ab}}$ & $1.56^{\mathrm{d}}$ & $\operatorname{tr}$ & - & $2.02^{\mathrm{k}}$ & $15.06^{\mathrm{s}}$ & $22.80^{\mathrm{i}}$ & $60.12^{\mathrm{m}}$ & $9.92^{r}$ \\
\hline $3 \mathrm{CO} 2$ & $12.14^{\mathrm{w}}$ & $3.49^{\mathrm{m}}$ & $24.53^{\mathrm{n}}$ & $55.01^{\mathrm{o}}$ & $0.77^{\mathrm{c}}$ & $0.26^{\mathrm{de}}$ & $0.10^{\mathrm{a}}$ & $1.10^{\mathrm{c}}$ & - & - & $2.60^{\mathrm{m}}$ & $15.89 \mathrm{t}$ & $24.63^{\mathrm{k}}$ & $56.88^{1}$ & $9.82^{\mathrm{p}}$ \\
\hline $\mathrm{HO} 1$ & $7.11^{\mathrm{n}}$ & $3.29^{1}$ & $13.42^{\mathrm{c}}$ & $54.57^{\mathrm{m}}$ & $19.32^{\mathrm{k}}$ & $0.85^{\mathrm{j}}$ & $0.44^{\mathrm{gh}}$ & $\operatorname{tr}$ & $\operatorname{tr}$ & $\operatorname{tr}$ & $1.00^{\mathrm{i}}$ & $11.25^{\circ}$ & $13.86^{\mathrm{c}}$ & $73.89^{t}$ & $6.09^{\mathrm{h}}$ \\
\hline $\mathrm{HO} 2$ & $6.17^{1}$ & $3.29^{1}$ & $14.46^{\mathrm{d}}$ & $54.77^{\mathrm{n}}$ & $18.71^{\mathrm{j}}$ & $1.08^{\mathrm{k}}$ & $0.50^{\mathrm{h}}$ & $\operatorname{tr}$ & $\operatorname{tr}$ & $\operatorname{tr}$ & $1.02^{\mathrm{i}}$ & $10.54^{\mathrm{n}}$ & $14.96^{\mathrm{d}}$ & $73.48^{\mathrm{s}}$ & $5.02^{\mathrm{e}}$ \\
\hline $\mathrm{EPO} 1$ & $5.57^{\mathrm{g}}$ & $2.49^{\mathrm{h}}$ & $10.88^{\mathrm{b}}$ & $64.09^{t}$ & $15.83^{\mathrm{i}}$ & $0.33^{\mathrm{f}}$ & $0.23^{\mathrm{de}}$ & - & - & - & $0.58^{\mathrm{e}}$ & $8.39^{\mathrm{g}}$ & $11.11^{\mathrm{b}}$ & $79.92^{\mathrm{u}}$ & $7.51^{\mathrm{k}}$ \\
\hline EPR2 & $6.51^{\mathrm{m}}$ & $1.76^{\mathrm{a}}$ & $8.65^{\mathrm{a}}$ & $73.54^{\times}$ & $8.72^{\mathrm{f}}$ & $0.31^{\mathrm{ef}}$ & $0.25^{\mathrm{ef}}$ & - & - & - & $0.26^{\mathrm{c}}$ & $8.58^{\mathrm{h}}$ & $8.90^{\mathrm{a}}$ & $82.26^{\mathrm{w}}$ & $6.93^{j}$ \\
\hline ИTO1 & $7.94^{\circ}$ & $5.52^{\mathrm{t}}$ & $25.72^{\circ}$ & $51.78^{\mathrm{j}}$ & $0.25^{\mathrm{ab}}$ & $3.59^{\circ}$ & $0.38^{\mathrm{g}}$ & - & - & - & $4.82^{\circ}$ & $17.05^{\mathrm{w}}$ & $26.10^{1}$ & $52.03^{\mathrm{f}}$ & $5.64^{\mathrm{f}}$ \\
\hline MTO2 & $8.16^{\mathrm{p}}$ & $5.54^{\mathrm{t}}$ & $23.33^{\mathrm{m}}$ & $53.87^{1}$ & $0.40^{\mathrm{b}}$ & $3.57^{\circ}$ & $1.02^{\mathrm{i}}$ & - & - & - & $4.11^{\mathrm{n}}$ & $17.27^{\times}$ & $24.35^{\mathrm{k}}$ & $54.27^{\mathrm{i}}$ & $5.86^{\mathrm{g}}$ \\
\hline POO1 & $9.05^{\mathrm{r}}$ & $2.23^{\mathrm{d}}$ & $17.73^{\mathrm{i}}$ & $69.24^{\mathrm{w}}$ & $0.97^{\mathrm{d}}$ & $0.16^{\mathrm{abc}}$ & $0.18^{\text {bcde }}$ & $\operatorname{tr}$ & - & $0.10^{\mathrm{a}}$ & $0.34^{\mathrm{cd}}$ & $11.44^{\mathrm{p}}$ & $18.01^{\mathrm{fg}}$ & $70.21^{\mathrm{p}}$ & $10.12^{\mathrm{s}}$ \\
\hline POO2 & $11.22^{\mathrm{t}}$ & $2.63^{\mathrm{ij}}$ & $19.65^{\mathrm{k}}$ & $62.88^{\mathrm{s}}$ & $1.21^{\mathrm{e}}$ & - & $0.20^{\text {cde }}$ & $\operatorname{tr}$ & - & $0.11^{\mathrm{a}}$ & $2.10^{\mathrm{k}}$ & $13.85^{\mathrm{r}}$ & $19.96^{\mathrm{h}}$ & $64.09^{\mathrm{n}}$ & $9.54^{\circ}$ \\
\hline PUO1 & $10.59^{\mathrm{s}}$ & $5.15^{\mathrm{s}}$ & $27.66^{\mathrm{p}}$ & $53.59^{\mathrm{k}}$ & $1.37^{\mathrm{e}}$ & $0.42^{\mathrm{g}}$ & $0.13^{\mathrm{abc}}$ & - & - & $\operatorname{tr}$ & $1.09^{\mathrm{i}}$ & $16.16^{\mathrm{u}}$ & $27.79^{\mathrm{m}}$ & $54.96^{\mathrm{j}}$ & $6.32 \mathrm{i}$ \\
\hline PUO2 & $11.28^{\mathrm{t}}$ & $5.99^{\mathrm{u}}$ & $35.69^{\mathrm{r}}$ & $44.95^{\mathrm{i}}$ & $0.18^{\mathrm{a}}$ & $0.47^{\mathrm{g}}$ & $0.11^{\mathrm{ab}}$ & - & - & $\operatorname{tr}$ & $1.33^{\mathrm{j}}$ & $17.74^{\mathrm{y}}$ & $35.80^{\mathrm{p}}$ & $45.13^{\mathrm{e}}$ & $6.07^{\mathrm{h}}$ \\
\hline SO1 & $3.42^{\mathrm{b}}$ & $2.40^{\mathrm{fg}}$ & $86.52^{\mathrm{w}}$ & $5.49^{\mathrm{a}}$ & $0.31^{\mathrm{ab}}$ & $0.25^{\mathrm{d}}$ & $0.32^{\mathrm{fg}}$ & $0.83^{\mathrm{b}}$ & $0.35^{\mathrm{a}}$ & - & $0.11^{\mathrm{b}}$ & $6.07^{\mathrm{b}}$ & $86.84^{\mathrm{u}}$ & $6.98^{\mathrm{a}}$ & $1.94^{\mathrm{a}}$ \\
\hline $\mathrm{SO} 2$ & $6.15^{1}$ & $4.25^{\mathrm{r}}$ & $18.52^{\mathrm{jk}}$ & $66.02^{\mathrm{u}}$ & $3.18^{f}$ & $0.26^{\mathrm{de}}$ & $0.10^{\mathrm{a}}$ & $0.86^{\mathrm{b}}$ & $0.35^{\mathrm{a}}$ & - & $0.32^{\mathrm{cd}}$ & $10.40^{\mathrm{n}}$ & $18.62^{\mathrm{g}}$ & $70.41^{\mathrm{p}}$ & $9.16^{\mathrm{m}}$ \\
\hline
\end{tabular}

Means within each column with different superscript letters $(\mathrm{a}-\mathrm{y})$ are significantly $(\alpha=0.05)$ different. Each value in the table represents the mean of three measurements

SFA saturated fatty acids, MUFA monounsaturated fatty acids, PUFA polyunsaturated fatty acids, $C O X$ calculated oxidizability value, $t r$ trace amounts $<0.10$ 
important, from the point of view of oil stability, is the content of polyunsaturated $\alpha$-linolenic acid. Linseed oils were the richest sources of the $\alpha$-linolenic acid (ALA). Also, $\alpha$ linolenic acid had the largest share in the fatty acid composition of investigated camelina seed oils' fatty acid composition of examined linseed oils was similar to those presented by Choo et al. (2007) - $\alpha$-linolenic acid content between 51.80 to $60.42 \%$ and Teh and Birch (2013)-59.34\%. Investigated camelina seed oils had a comparable fatty acid composition to oils analysed by Raczyk et al. (2016) and Ratusz et al. (2016).

Other tested oils showed the highest content of the linoleic acid. The maximum level of linoleic acid was reported for evening primrose oils. Also, poppy oils and sunflower seed oil (SO2) were characterised by high level of linoleic acid, at above $60 \%$. Hempseed, milk thistle and black cumin oils had a slightly lower content of this acid. A little higher level of linoleic acid in hempseed oil was recorded by Ten and Birch (2013) - 56.85\%. Similar results of fatty acid composition of milk thistle oils were presented by Fathi-Achachlouei and Azadmard-Damirchi (2009). Analysed black cumin oils' fatty acid composition differs from oils investigated by other researchers. Atta (2003) obtained a higher amount of linoleic acid (47.5\%) in black cumin oil. However, similar results were presented by Lutterodt et al. (2010) (58.83-61.20\%). Analysed pumpkin seed oils had different linoleic acid content; results did not differ from those presented by Vujasinovic et al. (2010) and Younis et al. (2000). The lowest content of linolenic acid was reported for linseed, rapeseed, camelina and one of sunflower oils.

\section{Oxidative Stability}

Oxidative stability is an important oil safety factor used to estimate its susceptibility to oxidation and consequently its shelf life. Primary products of the lipid oxidation are hydroperoxides, which result in the composition of short-chain and sensory-active compounds.

Results of analysed oils' oxidative stability determined using the Rancimat test are summarised in Table 3. The induction time of examined oils differed significantly. Black cumin oils followed by pumpkin seed oils were the most stable samples; their induction time was the longest. According to chemical dependence, chemical reaction time decreases two times if the reaction temperature rise to $10{ }^{\circ} \mathrm{C}$; black cumin oil (BCO2) oxidative stability was similar to those presented by Hassanien et al. (2014) - $16.9 \mathrm{~h}$ at $110^{\circ} \mathrm{C}$ and Gharby et al. (2015), $9-13 \mathrm{~h}$ at $110{ }^{\circ} \mathrm{C}$. However, a large variety of black cumin oil induction time was reported in the literature (Edris 2011). Examined pumpkin seed oils had higher induction time than oil analysed by Szterk et al. (2010)-13.63 h. Results of induction time presented by Neđeral et al. (2012) for husked pumpkin seed oil (23.8 h) and naked roasted seeds (30.3 h) were compatible with results for tested pumpkin oils. That
Table 3 Oxidative stability of analysed oils determined using the Rancimat and PDSC method

\begin{tabular}{|c|c|c|}
\hline Oil & Rancimat IP (h) & $\operatorname{PDSC} \tau_{\text {on }}(\min )$ \\
\hline LO1 & $3.84 \pm 0.09^{\mathrm{ab}}$ & $17.74 \pm 0.52^{\mathrm{abc}}$ \\
\hline LO2 & $4.48 \pm 0.10^{\mathrm{bc}}$ & $17.81 \pm 0.45^{\mathrm{abc}}$ \\
\hline LO3 & $4.65 \pm 0.11^{\mathrm{c}}$ & $19.35 \pm 0.32^{\mathrm{bcd}}$ \\
\hline LO4 & $3.87 \pm 0.07^{\mathrm{ab}}$ & $19.75 \pm 0.43^{\mathrm{bcd}}$ \\
\hline LO5 & $3.67 \pm 0.07^{\mathrm{a}}$ & $14.47 \pm 0.25^{\mathrm{a}}$ \\
\hline RO1 & $13.98 \pm 0.15^{\mathrm{h}}$ & $63.45 \pm 1.23^{\mathrm{k}}$ \\
\hline $\mathrm{RO} 2$ & $13.91 \pm 0.10^{\mathrm{gh}}$ & $54.65 \pm 1.01^{\mathrm{j}}$ \\
\hline $\mathrm{RO} 3$ & $13.76 \pm 0.04^{\mathrm{gh}}$ & $62.41 \pm 0.98^{\mathrm{k}}$ \\
\hline $\mathrm{RO} 4$ & $13.95 \pm 0.05^{\mathrm{h}}$ & $61.98 \pm 0.87^{\mathrm{k}}$ \\
\hline RO5 & $12.96 \pm 0.20^{\mathrm{g}}$ & $60.60 \pm 0.75^{\mathrm{k}}$ \\
\hline $\mathrm{CO} 1$ & $5.56 \pm 0.11^{\mathrm{d}}$ & $28.45 \pm 0.54^{\mathrm{f}}$ \\
\hline $\mathrm{CO} 2$ & $5.04 \pm 0.04^{\mathrm{cd}}$ & $17.75 \pm 0.34^{\mathrm{abc}}$ \\
\hline $\mathrm{CO} 3$ & $6.60 \pm 0.09^{\mathrm{e}}$ & $28.82 \pm 1.01^{\mathrm{f}}$ \\
\hline BCO1 & $13.45 \pm 0.10^{\mathrm{fgh}}$ & $20.64 \pm 0.32^{\text {cde }}$ \\
\hline $\mathrm{BCO} 2$ & $38.22 \pm 0.10^{\mathrm{k}}$ & $39.03 \pm 0.16^{\mathrm{hi}}$ \\
\hline EPO1 & $6.46 \pm 0.05^{\mathrm{e}}$ & $34.15 \pm 0.43^{\mathrm{g}}$ \\
\hline EPO2 & $6.35 \pm 0.10^{\mathrm{e}}$ & $34.60 \pm 0.64^{\mathrm{g}}$ \\
\hline $\mathrm{HO} 1$ & $5.43 \pm 0.05^{\mathrm{d}}$ & $15.19 \pm 0.32^{\mathrm{a}}$ \\
\hline $\mathrm{HO} 2$ & $6.59 \pm 0.08^{\mathrm{e}}$ & $22.62 \pm 0.65^{\mathrm{de}}$ \\
\hline MTO1 & $10.71 \pm 0.20^{\mathrm{f}}$ & $41.65 \pm 0.65^{\mathrm{i}}$ \\
\hline MTO2 & $5.47 \pm 0.06^{\mathrm{d}}$ & $16.34 \pm 0.54^{\mathrm{ab}}$ \\
\hline PO1 & $6.46 \pm 0.14^{\mathrm{e}}$ & $21.94 \pm 0.56^{\mathrm{de}}$ \\
\hline $\mathrm{PO} 2$ & $6.78 \pm 0.07^{\mathrm{e}}$ & $23.81 \pm 0.46^{\mathrm{e}}$ \\
\hline PUO1 & $20.29 \pm 0.20^{j}$ & $63.70 \pm 0.87^{\mathrm{k}}$ \\
\hline PUO2 & $34.39 \pm 0.29^{\mathrm{k}}$ & $78.39 \pm 0.92^{1}$ \\
\hline SFO1 & $19.87 \pm 0.07^{\mathrm{j}}$ & $102.84 \pm 0.91^{\mathrm{m}}$ \\
\hline SFO2 & $6.42 \pm 0.06^{\mathrm{i}}$ & $35.21 \pm 0.63^{\mathrm{gh}}$ \\
\hline
\end{tabular}

Each value in the table represents the mean of three measurements with standard deviation (SD) values obtained from analyses

Means within each column with superscript different letters $(a-n)$ are significantly $(\alpha=0.05)$ different

gives grounds to conclude that the analysed pumpkin oils have been produced from pre-treated seeds.

Less stable were cold-pressed rapeseed oils, obtained results did not differ from those presented by other researchers (Symoniuk et al. 2016). Oils from evening primrose, poppy and hemp seeds had more than twice lower oxidative stability compared to rapeseed oils. Furthermore, evening primrose and poppy seed oils were characterised by similar induction time determined by Rancimat method. Induction times of evening primrose oils did not differ from those presented by Szterk et al. (2010) - 6.34 h. Higher poppy oil induction time was obtained by Bozan and Temelli (2008) - $5.56 \mathrm{~h}$ at $110^{\circ} \mathrm{C}$. Hempseed oils' oxidative stability differs, which was also reported by Dimić et al. (2009) where oxidation induction time was between 6.4 to $7.4 \mathrm{~h}$. The induction period of camelina seed oils was similar to those presented by Szterk 
et al. (2010) - 6.32 $\mathrm{h}$ and Ratusz et al. (2016) — induction time was between 4.58 to $5.63 \mathrm{~h}$. Large differentiation of induction time was observed for milk thistle and sunflower oils. These discrepancies in the stability of sunflower oils may result from differences in their fatty acid composition. A high amount of oleic acid has been reported for sunflower oil (SFO1), which indicates that this oil was obtained from a high-oleic variety and was more stable.

Tested linseed oils were characterised by the lowest induction time determined using the Rancimat method. Analysed linseed oils' oxidative stability in Rancimat was shorter compared to the result obtained by Rudnik et al. (2001) - $6.40 \mathrm{~h}$, and Szterk et al. (2010) $-5.85 \mathrm{~h}$.

Evaluation of oils' oxidative stability was also performed using pressure differential scanning calorimetry (PDSC). The oxidative stability in PDSC method was marked as the onset time $\left(\tau_{\text {on }}\right)$, which corresponds with initiation stage of the oxidation process. Results of analysed oils' oxidative stability were presented in Table 3. According to obtained data, sunflower oil (SO1) was characterised by the highest induction time. Also, pumpkin seed oils were identified by high oxidative stability. The earliest onset induction time was marked for linseed oil was. Low oxidation time was also reported for hemp, poppy, camelina, milk thistle (MTO1) and black cumin seed oils. Linseed oil was more suitable for thermal oxidation, probably due to its high portion of PUFA. Hemp and poppy seed oils had a similar level of PUFA content, but the proportions of fatty acids C18:3 and C18:2 were different. In contrast to linseed oils, hemp and poppy seed oils were characterised by a higher content of C18:2 acid. The presence of antioxidants might also contribute to the differences in $\tau_{\text {on }}$. Black cumin oils, despite the same fatty acids composition, had different oxidative stability. The longer induction time of $\mathrm{BCO} 2$ might be influenced by great free radical scavenging capacity (3.18 mM of Trolox kg-1 oil). The same dependence occurred of sunflower and milk thistle oils.

\section{Statistical Analysis}

Since both methods are used to evaluate oils' oxidative stability, induction times as measured by the Rancimat and PDSC methods were compared using Pearson's linear correlation. The correlation coefficient of induction times was low and amounted $r=0.66$. Literature provides data that shows a high correlation between the Rancimat and PDSC method at different temperatures, but only for refined or cold-pressed oil from one type of raw material. Ciemniewska-Żytkiewicz et al. (2014) obtained a linear correlation of $>0.99$ for hazelnut oil, Symoniuk et al. (2016) for cold-pressed rapeseed and Ratusz et al. (2016), a polynomial correlation of $>0.98$ for camelina oil. On the other hand, results presented by Ramalho et al. (2011) have demonstrated no correspondence among the
Table 4 Correlation coefficient between oil chemical component and oxidative stability in Rancimat and PDSC test

\begin{tabular}{lcc}
\hline Quality factors & Rancimat IP & PDSC $\tau_{\text {on }}$ \\
\hline PV & $-0.49^{\mathrm{a}}$ & $-0.44^{\mathrm{a}}$ \\
$p$-AnV & 0.13 & 0.12 \\
Totox indicator & $-0.45^{\mathrm{a}}$ & $-0.40^{\mathrm{a}}$ \\
Chlorophylls & $0.62^{\mathrm{a}}$ & 0.05 \\
Carotenoids & $0.61^{\mathrm{a}}$ & 0.37 \\
SFA & 0.35 & -0.16 \\
MUFA & 0.36 & $0.80^{\mathrm{a}}$ \\
PUFA & $-0.46^{\mathrm{a}}$ & $-0.83^{\mathrm{a}}$ \\
C18:1 & $0.42^{\mathrm{a}}$ & $0.84^{\mathrm{a}}$ \\
C18:2 & 0.08 & -0.26 \\
C18:3 & $-0.54^{\mathrm{a}}$ & $-0.51^{\mathrm{a}}$ \\
COX & -0.38 & $-0.74^{\mathrm{a}}$ \\
TEAC & $0.43^{\mathrm{a}}$ & 0.12 \\
FAE & -0.06 & 0.35 \\
\hline
\end{tabular}

${ }^{\text {a }}$ Statistically significant correlation

PDSC and Rancimat method at one temperature for biodiesel from poultry fat oxidative stability evaluation.

As shown in Table 4, it might be concluded that oxidative stability as measured by the Rancimat method depends on many factors, but none of them characterised a high correlation. The highest dependencies were observed between the Rancimat induction time and the chlorophyll and carotenoid pigments content $(r=0.66)$. On the contrary, the content of

Table 5 Principal component analysis (PCA) factor loadings for the quality factors of analysed oils

\begin{tabular}{|c|c|c|c|c|c|}
\hline \multicolumn{3}{|l|}{ Rancimat method } & \multicolumn{3}{|l|}{ PDSC method } \\
\hline Quality factors & $\mathrm{PC} 1$ & $\mathrm{PC} 2$ & Quality factors & $\mathrm{PC} 1$ & $\mathrm{PC} 2$ \\
\hline PV & 0.385 & 0.255 & PV & 0.228 & 0.461 \\
\hline$p-\mathrm{AnV}$ & 0.004 & 0.006 & $p-\mathrm{AnV}$ & 0.003 & 0.006 \\
\hline TOTOX indicator & 0.349 & 0.227 & TOTOX indicator & 0.206 & 0.415 \\
\hline Chlorophylls & 0.050 & 0.462 & Chlorophylls & 0.000 & 0.434 \\
\hline Carotenoids & 0.103 & 0.266 & Carotenoids & 0.035 & 0.293 \\
\hline SFA & 0.000 & 0.734 & SFA & 0.058 & 0.661 \\
\hline MUFA & 0.638 & 0.347 & MUFA & 0.835 & 0.138 \\
\hline PUFA & 0.738 & 0.214 & PUFA & 0.877 & 0.056 \\
\hline C18:1 & 0.727 & 0.235 & $\mathrm{C} 18: 1$ & 0.892 & 0.062 \\
\hline C18:2 & 0.020 & 0.609 & $\mathrm{C} 18: 2$ & 0.103 & 0.569 \\
\hline C18:3 & 0.450 & 0.154 & $\mathrm{C} 18: 3$ & 0.306 & 0.335 \\
\hline $\operatorname{cox}$ & 0.567 & 0.017 & COX & 0.617 & 0.010 \\
\hline FAE & 0.071 & 0.000 & FAE & 0.092 & 0.017 \\
\hline TEAC & 0.064 & 0.002 & TEAC & 0.028 & 0.001 \\
\hline Rancimat IP & 0.536 & 0.173 & $\operatorname{PDSC} \tau_{\text {on }}$ & 0.873 & 0.006 \\
\hline
\end{tabular}

Values in italic correspond for each variable to the factor for which the squared cosine is the largest 
oleic $(r=0.84)$, monounsaturated $(r=0.80)$ and polyunsaturated $(r=-0.83)$ fatty acids had the greatest impact on the oxidative stability of cold-pressed oils as measured by the PDSC method. Secondary oxidation products, saturated fatty acids and C18:2 content do not affect the oxidative stability of oil as determined using both methods. The detected regularities and correlation between quality characteristics and oxidative stability was verified by applying the analysis of the main components. PCA was performed for the mean ratings of oils across the 16 attributes (Table 5). As presented in Fig. 1, the first two PCs for the Rancimat method accounted for $56.02 \%$ of variability $(31.34$ and $24.68 \%$, respectively). The following quality features contributed highly to PC1: PUFA (0.738) and C18:1 (0.727) while saturated fatty acids (SFA) (0.734) and 18:3 (0.609) contributed highly to PC2 Table 5. PCA biplot for PDSC method shows that two first to PCs accounted for 57.46\%. C18:1 (0.892), PUFA (0.877), PDSC $\tau_{\text {on }}(0.873)$ and MUFA (0.835) were highly contributed to PC1, while SFA (0.569) and C18:1 (0.661) contributed highly to PC2.
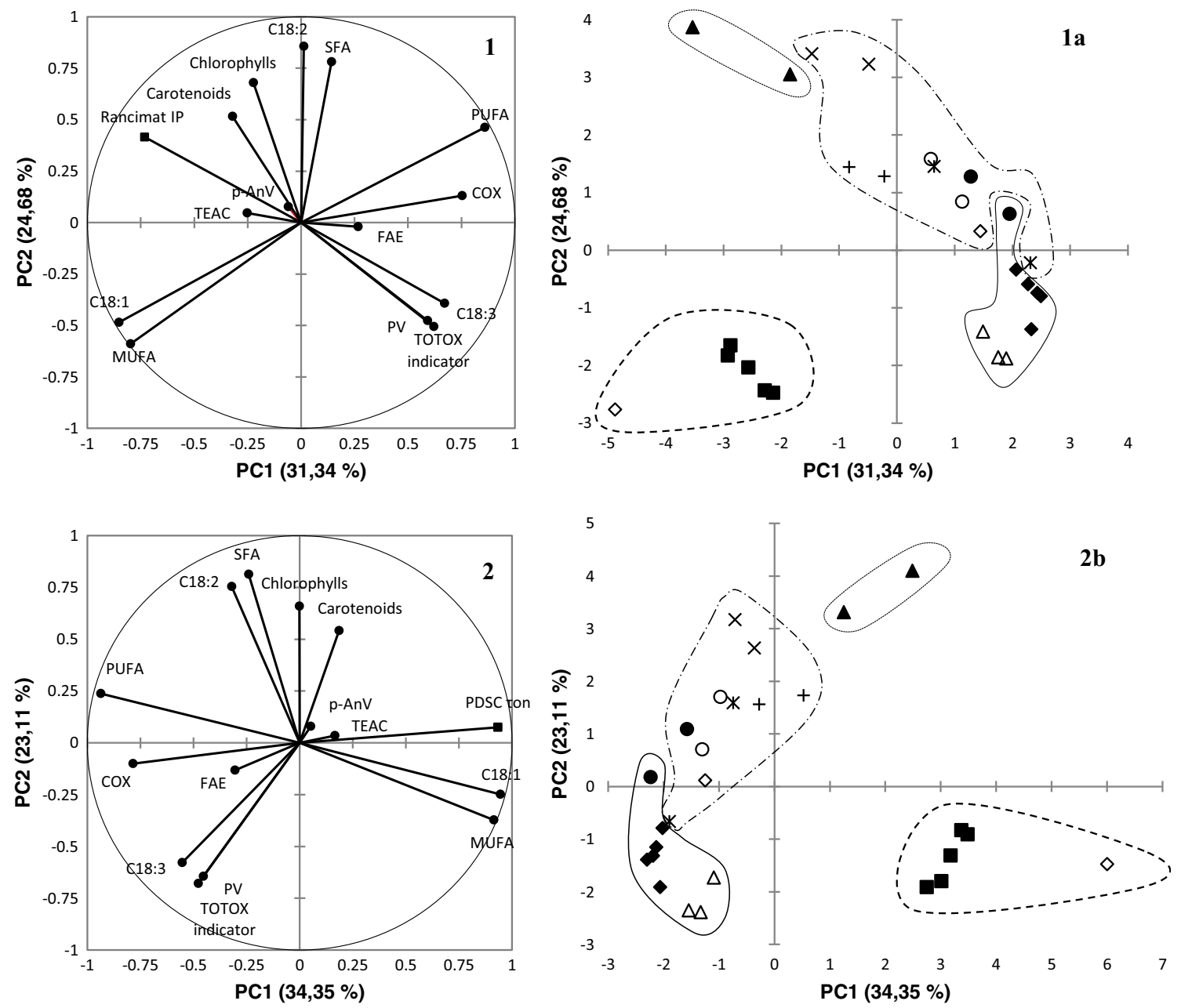

Fig. 1 Loading and score plots of PC1-PC2 for different cold-pressed oils and divided into oxidation stability assessment method. (1) Rancimat and (2) PDSC method

Oil symbols (1a, 1b): - - LO, $-\mathrm{RO}, \triangle-\mathrm{CO}, \times-\mathrm{BCO}, \square-\mathrm{EPO}, \bullet-\mathrm{HO},+-\mathrm{MTO}, \circ-\mathrm{PO}, \boldsymbol{\Delta}-\mathrm{PUO}, \diamond-\mathrm{SO}$. 
thistle, poppy oils and one of sunflower (SO2) and hempseed (HO2) oils represented the last four groups. However, it should be noted that depending on the oxidative stability assessing method, oil groups did not differ.

\section{Conclusions}

The results of this study showed that both accelerated methods, the Rancimat and pressure differential scanning calorimetry, used to assess oxidation stability of cold-pressed oils from different oilseeds, were not highly correlated $(r=0.66)$. Both oxidative stability evaluating methods should not be used interchangeably to compare the stability of the various cold-pressed oils. Induction time measured using the Rancimat and PDSC methods depend on the tested features to varying degree. PCA demonstrated that none of the selected quality characteristics influenced strongly on tested coldpressed oils oxidative stability determined by the Rancimat test. In contrast to Rancimat method, cold-pressed oils induction times in PDSC mainly depend on the fatty acids composition. The methods used to assess the stability of oils did not significantly affect the division of oils in terms of their overall quality. This proves that oil quality should be considered not only on the basis of their oxidative stability but all qualities should be taken into account.

\section{Compliance with Ethical Standards}

Conflict of Interest Edyta Symoniuk declares that she has no conflict of interest. Katarzyna Ratusz declares that she has no conflict of interest. Ewa Ostrowska-Ligęza declares that she has no conflict of interest. Krzysztof Krygier declares that he has no conflict of interest.

Ethical Approval This article does not contain any studies with human participants or animals performed by any of the authors.

Open Access This article is distributed under the terms of the Creative Commons Attribution 4.0 International License (http:// creativecommons.org/licenses/by/4.0/), which permits unrestricted use, distribution, and reproduction in any medium, provided you give appropriate credit to the original author(s) and the source, provide a link to the Creative Commons license, and indicate if changes were made.

\section{References}

AOAC Official Method 996.06 (2001) Fat (Total, Saturated, and Unsaturated) in Foods. Hydrolytic extraction gas chromatographic method

AOCS Method Cc 13i-96 (1998) Official methods and recommended practice of the American Oil Chemists Society, 5th edn. AOCS Press, Champaign

AOCS Official Method 965.33 (1999) Peroxide value of oils and fats

AOCS Official Method Cd 18-20 (2002) p-Anisidine value

AOCS Official Method Cd 3d-63 (2000) Acid value
AOCS Official Method Ce 2-66 (1997) Preparations of methyl esters of fatty acids

Atta MB (2003) Some characteristics of nigella (Nigella sativa L.) seed cultivated in Egypt and its lipid profile. Food Chem 83:63-68

Bozan B, Temelli F (2008) Chemical composition and oxidative stability of flax, safflower and poppy seed and seed oils. Bioresour Technol 99:6354-6359

BS 684-2,20 (1977) Methods of analysis of fats and fatty oils. Other methods. Determination of carotene in vegetable oils

Choe E, Min DB (2006) Mechanisms and factors for edible oil oxidation. Compr Rev Food Sci Food Saf 5:169-186

Choo WS, Birch J, Dufour JP (2007) Physicochemical and quality characteristics of cold-pressed flaxseed oils. J Food Comp Anal 20:202211

Ciemniewska-Żytkiewicz H, Ratusz K, Bryś J, Reder M, Koczoń P (2014) Determination of the oxidative stability of hazelnut oils by PDSC and Rancimat methods. J Therm Anal Calorim 118:875-881

Dimić E, Romanić R, Vujasinović V (2009) Essential fatty acids, nutritive value and oxidative stability of cold pressed hempseed (Cannabis sativa L.) oil from different varieties. Acta Aliment 38:229-236

Edris AE (2011) The chemical composition and the content of volatile oil: potential factors that can contribute to the oxidative stability of Nigella sativa L. crude oil . J Diet Suppl 8:34-42

Espin JC, Soler-Rivas C, Wichers HJ (2000) Characterization of the total free radical scavenger capacity of vegetable oils and oil fractions using 2, 2-diphenyl-1-picrylhydrazyl radical. J Agric Food Chem 48:648-656

Farhoosh R (2007) The effect of operational parameters of the Rancimat method on the determination of the oxidative stability measures and shelf-life prediction of soybean oil. J Am Oil Chem Soc 84:205-209

Fatemi SH, Hammond EG (1980) Analysis of oleate, linoleate and linolenate hydroperoxides in oxidized ester mixtures. Lipids 15: 379-385

Fathi-Achachlouei B, Azadmard-Damirchi S (2009) Milk thistle seed oil constituents from different varieties grown in Iran. J Am Oil Chem Soc 86:643-649

Gharby S, Harhar H, Guillaume D, Roudani A, Boulbaroud S, Ibrahimi M, Ahmad M, Sultana S, Hadda TB, Chafchaouni-Moussaoui I, Charrouf Z (2015) Chemical investigation of Nigella sativa L. seed oil produced in Morocco. J Saudi Soc Agricultural Sci 14:172-177

Górnaś P, Siger A, Juhņeviča K, Lācis G, Šnē E, Segliņa D (2014) Coldpressed Japanese quince (Chaenomeles japonica (Thunb.) Lindl. ex Spach) seed oil as a rich source of $\alpha$-tocopherol, carotenoids and phenolics: a comparison of the composition and antioxidant activity with nine other plant oils. Eur J Lipid Sci Technol 116:563-570

Haron H, Grace-Lynn C, Shahar S (2014) Comparison of physicochemical analysis and antioxidant activities of Nigella sativa seeds and oils from Yemen, Iran and Malaysia. Sains Malaysiana 43:535-542

Hassanien MM, Abdel-Razek AG, Rudzińska M, Siger A, Ratusz K, Przybylski R (2014) Phytochemical contents and oxidative stability of oils from non-traditional sources. Eur J Lipid Sci Technol 116: $1563-1571$

Kamal-Eldin A (2006) Effect of fatty acids and tocopherols on the oxidative stability of vegetable oils. Eur J Lipid Sci Technol 108:1051-1061

Kowalski B, Ratusz K, Kowalska D, Bekas W (2004) Determination of the oxidative stability of vegetable oils by differential scanning calorimetry and Rancimat measurements. Eur J Lipid Sci Technol 106: $165-169$

Lutterodt H, Luther M, Slavin M, Yin JJ, Parry J, Gao JM, Yu LL (2010) Fatty acid profile, thymoquinone content, oxidative stability, and antioxidant properties of cold-pressed black cumin seed oils. Food Sci Technol 43:1409-1413

Matthäus B, Brühl L (2003) Quality of cold-pressed edible rapeseed oil in Germany. Food/Nahrung 47:413-419 
Neđeral S, Škevin D, Kraljić K, Obranović M, Papeša S, Bataljaku A (2012) Chemical composition and oxidative stability of roasted and cold pressed pumpkin seed oils. J Am Oil Chem Soc 89:1763-1770

Prescha A, Grajzer M, Dedyk M, Grajeta H (2014) The antioxidant activity and oxidative stability of cold-pressed oils. J Am Oil Chem Soc 91:1291-1301

Raczyk M, Popis E, Kruszewski B, Ratusz K, Rudzińska M (2016) Physicochemical quality and oxidative stability of linseed (Linum usitatissimum) and camelina (Camelina sativa) cold-pressed oils from retail outlets. Eur J Lipid Sci Technol 118:834-839

Ramalho EFSM, Albuquerque AR, Souza AL, Barro AK, Maia AS, Santos IMG, Souza AG (2011) Use of different techniques in the evaluation of the oxidative stability of poultry fat biodiesel. J Therm Anal Calorim 106:787-791

Ratusz K, Popis E, Ciemniewska-Żytkiewicz H, Wroniak M (2016) Oxidative stability of camelina (Camelina sativa L.) oil using pressure differential scanning calorimetry and Rancimat method. J Therm Anal Calorim 126:343-351

Rice-Evans CA, Miller NJ, Bolwell PG, Bramley PM, Pridham JB (1995) The relative antioxidant activities of plant-derived polyphenolic flavonoids. Free Radic Res 22:375-383

Rudnik E, Szczucinska A, Gwardiak H, Szulc A, Winiarska A (2001) Comparative studies of oxidative stability of linseed oil. Thermochim Acta 370:135-140

Sánchez-Moreno C, Larrauri JA, Saura-Calixto F (1998) A procedure to measure the antiradical efficiency of polyphenols. J Sci Food Agric 76:270-276

Shahidi F, Zhong Y (2005) Lipid oxidation: measurement methods. In: Bailey's industrial oil and fat products. Wiley, New York, pp 1-8

Shahidi F, Zhong Y (2010) Lipid oxidation and improving the oxidative stability. Chem Soc Rev 39:4067-4079
Sielicka M, Małecka M, Purłan M (2014) Comparison of the antioxidant capacity of lipid-soluble compounds in selected cold-pressed oils using photochemiluminescence assay (PCL) and DPPH method. Eur J Lipid Sci Technol 116:388-394

Siger A, Nogala-Kałucka M, Lampart-Szczapa M (2008) The content and antioxidant activity of phenolic compounds in cold-pressed plant oils. J Food Lipids 15:137-149

Symoniuk E, Ratusz K, Krygier K (2016) Comparison of the oxidative stability of cold-pressed rapeseed oil using pressure differential scanning calorimetry and Rancimat methods. Eur J Lipid Sci Technol. https://doi.org/10.1002/ejlt.201600182

Szterk A, Roszko M, Sosińska E, Derewiaka D, Lewicki PP (2010) Chemical composition and oxidative stability of selected plant oils. J Am Oil Chem Soc 87:637-645

Šimon P, Kolman L' (2001) DSC study of oxidation induction periods. J Therm Anal Calorim 64:813-820

Šimon P, Kolman Ĺ, Niklová I, Schmidt Š (2000) Analysis of the induction period of oxidation of edible oils by differential scanning calorimetry. J Am Oil Chem Soc 77:639-642

Teh SS, Birch J (2013) Physicochemical and quality characteristics of cold-pressed hemp, flax and canola seed oils. J Food Comp Anal 30:26-31

Vujasinovic V, Djilas S, Dimic E, Romanic R, Takaci A (2010) Shelf life of cold-pressed pumpkin (Cucurbita pepo L.) seed oil obtained with a screw press. J Am Oil Chem Soc 87:1497-1505

Woo L, Khare A, Sandford C, Ling M, Ding S (2001) Relevance of high temperature oxidative stability testing to long term polymer durability. J Therm Anal Calorim 64:539-548

Younis YMH, Ghirmay S, Al-Shihry SS (2000) African Cucurbita pepo L.: properties of seed and variability in fatty acid composition of seed oil. Phytochemistry 54:71-75 